\title{
METHODS IN BRIEF
}

\section{EPIGENETICS}

\section{Low-input ChIP for immunogenomics}

Specific histone modifications are associated with the position and activity of regulatory regions in the genome and can be localized using chromatin immunoprecipitation (ChIP). However, ChIP requires a large number of cells, whereas many dynamic biological events involve small, transient cell populations. Lara-Astiaso et al. developed an indexing-first ChIP (iChIP) procedure in which total chromatin from individual fixed and cell-sorted samples is ligated to DNA barcodes. iChIP avoids low-input enzymatic steps and is compatible with large-scale pooling, making it sensitive and reproducible even from just a few hundred cells. The authors used iChIP to characterize the dynamics of thousands of enhancer and promoter regions by following four chromatin modifications along 16 welldefined steps of hematopoietic differentiation. They identified tens of thousands of de novo lineage-specific enhancers and used gene expression profiling to relate chromatin dynamics to a transcription factor network.

Lara-Astiaso, D. et al. Science 345, 943-949 (2014).

\section{IMAGING}

\section{Whole organs and animals rendered transparent}

Organs such as the brain, kidney or lungs depend on a complex three-dimensional organization for their function. Yang et al. now describe an improved tissue 'clearing' protocol that preserves structural integrity even at the cellular level while enabling the imaging of structures deep inside the tissue. The approach combines an optimized set of clearing reagents, including acrylamide and a strong detergent, with a suitable embedding medium. The tissue clearing process can be sped up by perfusing the clearing agents into organs or even whole animals. The procedure is compatible with histological techniques, immunohistochemistry and fluorescence in situ hybridization and can be applied to a variety of organs. The approach allows tissues and organs to be studied in their intact structural context.

Yang, B. et al. Cell 158, 945-958 (2014).

\section{SEQUENCING}

\section{Sampling local sequence with proximity ligation}

Targeted resequencing methods are widely used, but these techniques do not discriminate between alleles and typically require that much of the target sequence be known. de Vree et al. exploit the observation that proximity ligation-based assays such as chromosome conformation capture on-chip (4C) are highly enriched for neighboring DNA sequences. Their new targeted locus amplification (TLA) method begins with a single anchor sequence and, unlike $4 C$, does not enrich for contact points after a second digestion step. Mainly using the BRCA genes, the authors demonstrate TLA for resequencing tens to hundreds of kilobases, haplotyping, assessing genetic and structural variation, and sequencing transgene integration sites.

de Vree, P.J.P. et al. Nat. Biotechnol. doi:10.1038/nbt.2959 (17 August 2014).

\section{GENOMICS}

\section{Compressed interaction networks assign gene function}

Assigning function to genes remains a big challenge in biology. Researchers often turn to gene or protein networks to infer the role of a particular gene. Local network analysis identifies the function of a network node by existing annotations, which can be insufficient. Global network analysis looks for relatedness over an entire network and is therefore more sensitive, but it is also more computationally expensive and thus restricted to networks of no more than several thousand nodes. Lisewski et al. circumvent this limitation by using a compression scheme that eliminates redundancies between and within networks. This allowed them to analyze networks of genes from hundreds of genomes. As demonstration, they identified the function of a Plasmodium falciparum antigen with potential as a drug target.

Lisewski, A.M. et al. Cell 158, 916-928 (2014). 